\title{
Biologia reprodutiva de Campylocentrum micranthum (Orchidaceae, Angraecinae)
}

Reproductive biology of Campylocentrum micranthum (Orchidaceae, Angraecinae)

\author{
Paulo Roberto de Medeiros Cabral ${ }^{1,3}$ \& Emerson Ricardo Pansarin ${ }^{2}$
}

\begin{abstract}
Resumo
Campylocentrum micranthum é uma espécie amplamente distribuída pelo Brasil. A biologia reprodutiva de C. micranthum foi estudada no município de São Simão, no noroeste do estado de São Paulo. A vegetação da região é caracterizada por florestas semideciduais, matas palustres e cerrado. Na área de estudo $C$. micranthum floresce no verão. A população estudada é formada por mais de 35 indivíduos distribuídos em uma área de mata palustre. As plantas são epífitas, e as flores são ressupinadas e de coloração creme. Cada flor possui um nectário tubuloso na base que produz em média 0,053 $\mu \mathrm{L}$ de néctar. As atividades dos polinizadores se iniciam por volta das 07:30 e as visitas se estendem até 13:00 h. Os polinizadores de $C$. micranthum são abelhas dos gêneros Lophopedia e Ceratina. A espécie autocompatível e não autógama. No entanto, necessita de polinizadores para a transferência de pólen. Nos experimentos de polinização cruzada à formação de frutos foi de $3,17 \%$, enquanto no tratamento de autopolinização manual foi obtido $16 \%$ de frutificação. Em condições naturais a taxa de frutificação foi de $36,4 \%$.
\end{abstract}

Palavras-chave: Angraecinae, biologia reprodutiva, Campylocentrum, Orchidaceae, polinização.

\begin{abstract}
Campylocentrum micranthum is widely distributed throughout Brazil. The reproductive biology of $C$. micranthum was studied in the municipality of São Simão, state of São Paulo, Brazil. The vegetation of the region is characterized by semi-deciduous forests, swamp forests and cerrado. In the study area, $C$. micranthum blooms in summer. The study population consists of more than 35 individuals distributed in a swamp forest area. The plants are epiphytes and the flowers are resupinate and creamy in colour. Each flower has a tubular nectary at the base of the labellum that produces $0.053 \mu \mathrm{L}$ of nectar on average. The activity of the pollinator on flowers occurs from 07:30 to 13:00. The pollinators of C. Micrantum are bees of the genera Lophopedia and Ceratina. Campylocentrum micranthum is self-compatible, but dependent on a biotic pollinator for pollen transference. In the treatment of cross-pollination, fruit set was $3.17 \%$, while in the experiment of manual self-pollination $16 \%$ fructification was recorded. Under natural conditions (open pollination) fruit set was $36.4 \%$.
\end{abstract}

Key words: Angraecinae, reproductive biology, Campylocentrum, Orchidaceae, pollination.

\section{Introdução}

Angraecinae Summerh. é uma subtribo dentro da subfamília Epidendroideae com cerca de 18 gêneros e aproximadamente 408 espécies (Dressler 1993). Entre esses gêneros está Campylocentrum Benth., com cerca de 60 espécies distribuídas pelo México até o sul do Brasil, Bolívia e Caribe (Todzia 1980; Carlsward et al. 2003), sendo um dos gêneros de orquídeas mais heterogêneos em relação à morfologia vegetativa (Hoehne 1949). O Brasil possui 36 espécies de Campylocentrum (Barros et al.2012), e a região sul do país é considerada como o principal centro de distribuição para o gênero (Todzia 1980; Carlsward et al. 2003). No interior do estado de São Paulo o gênero está representado por 10 espécies, incluindo Campylocentrum micranthum (Lindl.) Rolfe (Pansarin \& Pansarin 2008,2010; Ferreira et al. 2010). Campylocentrum

\footnotetext{
${ }^{1}$ Universidade de São Paulo, Programa de Pós-graduação em Entomologia, FFLCRP, Depto. Biologia, Av. Bandeirantes 3900, 14040-901, Ribeirão Preto, SP, Brasil.

${ }^{2}$ Universidade de São Paulo, FFLCRP, Depto. Biologia, Lab. Biologia Molecular e Biossistemática de Plantas, Av. Bandeirantes 3900, 14040-901, Ribeirão Preto, SP, Brasil.

${ }^{3}$ Autor para correspondência: pracabral@gmail.com
} 
micranthum está amplamente distribuída pelo Brasil, não ocorrendo apenas na região sul do país (Pabst \& Dungs 1977; Pansarin \& Pansarin 2010; Barros et al. 2012).

Estudos sobre polinização de Campylocentrum são escassos. No entanto, de acordo com Bogarín \& Pupulin (2010), as flores alaranjadas, amarelas ou rosas, a produção de fragrâncias, a presença de nectários, entre outras características, comuns em muitas espécies desse grupo sugerem polinização por agentes bióticos, incluindo micro lepidópteros e abelhas. Segundo Van der Pijl \& Dodson (1966), os Himenópteros, principalmente as abelhas, têm sido reconhecidos como o grupo mais importante de polinizadores em Orchidaceae, sendo responsável pela polinização de cerca de $60 \%$ das espécies de orquídeas. Estudos realizados por Singer \& Coccuci (1999) no sul do Brasil indicam que as flores de Campylocentrum aromaticum Barb. Rodr. são polinizadas por abelhas Halictidae e C. burchellii Cogn. é polinizado por abelhas da tribo Meliponini. $\mathrm{Na}$ Costa Rica, Borgarín \& Pupulin (2010), em um estudo com Campylocentrum sp., não observaram polinizadores para espécie. Segundo Damon \& Salas-Reblero (2007), em um estudo realizado no México, observaram que Campylocentrum micranthum se reproduz por autogamia, sendo observado alto índice de frutificação em condições naturais. No Brasil, até onde é conhecido, não existe nenhum estudo sobre a biologia reprodutiva envolvendo C. micranthum.

O presente trabalho teve como objetivos estudar a biologia reprodutiva de Campylocentrum micranthum, analisando os aspectos da fenologia, morfo-anatomia floral, seus polinizadores e mecanismos de polinização, bem como o sucesso reprodutivo da espécie em condições naturais.

\section{Material e Métodos}

A biologia reprodutiva de Campylocentrum micranthum (Lindl.) Rolfe foi estudada no município de São Simão (aproximadamente $21^{\circ} 29^{\prime} \mathrm{S}$ e $47^{\circ} 36^{\prime} \mathrm{O}$ ), no noroeste do estado de São Paulo, sudeste do Brasil. A vegetação da região é caracterizada por florestas estacionais semideciduais, matas palustres, cerrado e campocerrado. As áreas de vegetação nativa da região vêm sofrendo constantes intervenções antrópicas relacionadas aos ciclos econômicos de extração de areia, agricultura e pecuária. O município de São Simão é caracterizado por altitudes que variam entre 620 e $700 \mathrm{~m}$ com temperaturas médias mensais entre $17,6{ }^{\circ} \mathrm{C}$ no mês mais frio (julho), e $23,5^{\circ} \mathrm{C}$ no mês mais quente (fevereiro). A precipitação média anual é de $1478 \mathrm{~mm}$ (CEPAGRI 2012). O clima da região enquadra-se na categoria "Cwa", segundo a classificação de Köppen (1948), ou seja, temperado, macrotérmico, moderadamente chuvoso e com inverno seco não rigoroso. A população estudada é formada por mais de 35 indivíduos distribuídos em uma área de mata palustre.

Para acompanhamento da fenologia de Campylocentrum micranthum foram realizadas visitas mensais a população de estudo, entre outubro de 2010 e outubro de 2011, para verificação da produção e desenvolvimento das inflorescências, período de antese, fenecimento das flores e deiscência dos frutos. As observações sobre o período de antese foram realizadas no período entre as $7 \mathrm{~h}$ e $18 \mathrm{~h}$.

Os estudos sobre morfologia floral foram realizados utilizando 30 flores frescas. Suas estruturas foram observadas utilizando um microscópio estereoscópico binocular e medidas com um paquímetro.

Para caracterizar a estrutura anatômica das áreas produtoras de recursos, flores foram fixadas em FAA $50 \%$ e FNT por 48 h e estocadas em etanol $70 \%$. Posteriormente as flores foram desidratadas em série butílica (Johansen 1940), incluídas em parafina e seccionadas (transversal e longitudinalmente) em micrótomo rotativo (cortes com espessura entre 10-12 $\mu \mathrm{m}$ ). Os cortes foram corados com Safranina O e Azul de Astra (Gerlach 1969) e as lâminas permanentes foram montadas com resina sintética.

Para calcular o volume do néctar foram obtidas medidas da coluna do néctar acumulado no interior do nectário de 30 flores, utilizando a fórmula universal para cálculo de cilindro $\mathrm{V}=$ $\pi \cdot \mathrm{r}^{2} . \mathrm{h}$, onde $\mathrm{h}=$ altura da coluna de néctar e $\mathrm{r}=$ raio do lúmen do nectário.

As observações dos visitantes florais e dos mecanismos de polinização de Campylocentrum micranthum foram realizadas no município de São Simão, entre 14-19 e 21-27 de janeiro de 2011, totalizando 94 horas de observação. O período de observação entre os dias14-19 aconteceu das 7 h $30 \mathrm{~min}$ às $17 \mathrm{~h} 30 \mathrm{~min}$, enquanto entre os dias 21-27 as observações ocorreram das 7 h $30 \mathrm{~min}$ às $14 \mathrm{~h} 30 \mathrm{~min}$. Para detectar a possível ocorrência de polinizadores noturnos flores $(n=30)$ foram marcadas ao final de cada dia de observação e conferidas na manhã do dia seguinte. 
Os tratamentos para verificação do sistema reprodutivo de Campylocentrum micranthum foram realizados a partir de indivíduos coletados na área de estudo e cultivados no Orquidário do Laboratório de Biologia Molecular e Biossistemática de Plantas (LBMBP) da Faculdade de Filosofia, Ciências e Letras, USP, Ribeirão Preto, estado de São Paulo, Brasil $\left(21^{\circ} 10^{\prime} \mathrm{S}\right.$ e $\left.47^{\circ} 48^{\prime} \mathrm{O}\right)$. Os indivíduos foram coletados em diferentes forófitos. Foram utilizados 30 indivíduos, os quais foram submetidos aos tratamentos de autopolinização manual, polinização cruzada e emasculação (teste para apomixia), além de observações sobre a ocorrência de autopolinização espontânea, sendo utilizadas de duas a três inflorescências de cada indivíduo nos tratamentos, que foram feitos a partir de flores do primeiro dia de antese. $\mathrm{O}$ número de flores usadas em cada tratamento foi de 50 para autopolinização manual, 30 para emasculação e 63 para polinização cruzada. Para os testes sobre polinização espontânea foram usadas 82 flores. As repetições foram realizadas de forma que todos os indivíduos utilizados pudessem receber todos os tipos de tratamentos. Devido ao tamanho muito reduzido das flores os tratamentos foram realizados sob o microscópio estereoscópico binocular. Para que isso fosse possível, as plantas foram removidas dos vasos e replantadas após as manipulações. Os indivíduos utilizados nos tratamentos foram mantidos no orquidário que apresenta toda estrutura revestida com tela $50 \%$ de sombreamento que funciona como barreira contra ação de eventuais visitantes florais. As informações sobre a taxa de desenvolvimento de frutos em condições naturais (polinização aberta) foram obtidas no município de São Simão, em março de 2011. Foram utilizados 30 indivíduos contando-se o número de flores produzidas e o número de frutos desenvolvidos. Um indivíduo Campylocentrum micranthum foi coletado como material testemunho (Brasil, São Paulo, São Simão, 24/I/2010, Col. E.R. Pansarin, LBMBP 460) e depositado no Orquidário do Laboratório de Biologia Molecular e Biossistemática de Plantas $(<\mathrm{http}$ ://splink.cria.org.br/manager/ detail? setlang $=$ pt\&resource $=$ LBMBP $>$ ). Os insetos foram coletados utilizando uma rede entomológica, para posterior identificação e depositado como material testemunho na Coleção Entomológica da Universidade de Brasília (UNB).

\section{Resultados}

$\mathrm{Na}$ área estudada os indivíduos de Campylocentrum micranthum florescem no verão (dezembro-fevereiro). A maioria dos indivíduos florescem entre o final de dezembro e janeiro, com alguns poucos florescendo em fevereiro. As plantas são epífitas, monopodiais e produzem até seis inflorescências laterais. As inflorescências são racemosas e cada uma produz até 12 flores (Fig. 1a). As flores são ressupinadas de coloração predominantemente creme. A sépala dorsal (ca. 3-4 $\times 1 \mathrm{~mm}$ ) é elíptica e as laterais medem ca. 3-4,5 $\times 1 \mathrm{~mm}$ e são lanceoladas a elíptico-lanceoladas com ápice acuminado. As pétalas (ca. 3-4×1 mm) são lanceoladas. O labelo é 3-lobado (ca. 3-3,5 $\times 1 \mathrm{~mm}$ ), e de coloração marrom do meio para a base e branco em direção ao ápice. A coluna é branca, mede ca. $1 \times 1 \mathrm{~mm}$, apresenta estigma (ca. $0,4 \mathrm{~mm}$ diâm.) circular e antera (ca. 0,4-0,5 mm compr.) oval. O polinário (ca. $0,5 \mathrm{~mm}$ compr.) é formado por duas polínias ovais, amarelas e que medem ca. $0,2 \mathrm{~mm}$ compr. Na base do labelo há um nectário cilíndrico e que produz em média $0,053 \mu \mathrm{L}$ $(0,046-0,061 \mu L)$ de néctar (Figs. 1b,c). O néctar é secretado pelas células epidérmicas da parede interna do nectário, que é lisa, não apresentando tricomas ou papilas. As células do parênquima nectarífero tiveram o protoplasto bem corado, que possivelmente também estejam envolvidas no processo de secreção (Figs. 1d,e). As flores de Campylocentrum micranthum apresentam antese diurna e se abrem em sucessão, da base para o ápice da inflorescência. A cada dia 4 a 6 flores iniciam a antese. A cada flor intacta dura de oito a nove dias.

As flores de Campylocentrum micranthumforam visitadas e polinizadas por abelhas do gênero Lophopedia pygmaea Schrottky 1902, L. nigrispinis Vachal 1909 (Apidae, Tapinotaspidini; Fig. 1f), e Ceratina sp. (Apidae, Xylocopinae). As atividades dos polinizadores às flores se iniciam por volta das $7 \mathrm{~h} 30 \mathrm{~min}$ e se estendem até às $13 \mathrm{~h}$. As visitas ocorreram apenas em dias ensolarados. Cada abelha visitou de uma a quatro flores por inflorescência, e cada uma delas com duração de cerca de 1-8 segundos. A visita se iniciou com a abelha pousando sobre a inflorescência. Em seguida, a abelha introduziu a glossa no nectário para acessar o néctar. Nesse momento a porção dorsal das estruturas entra em contato com o viscídio do polinário, que é removido, ficando preso as peças bucais das abelhas. O polinário foi removido com ou sem 

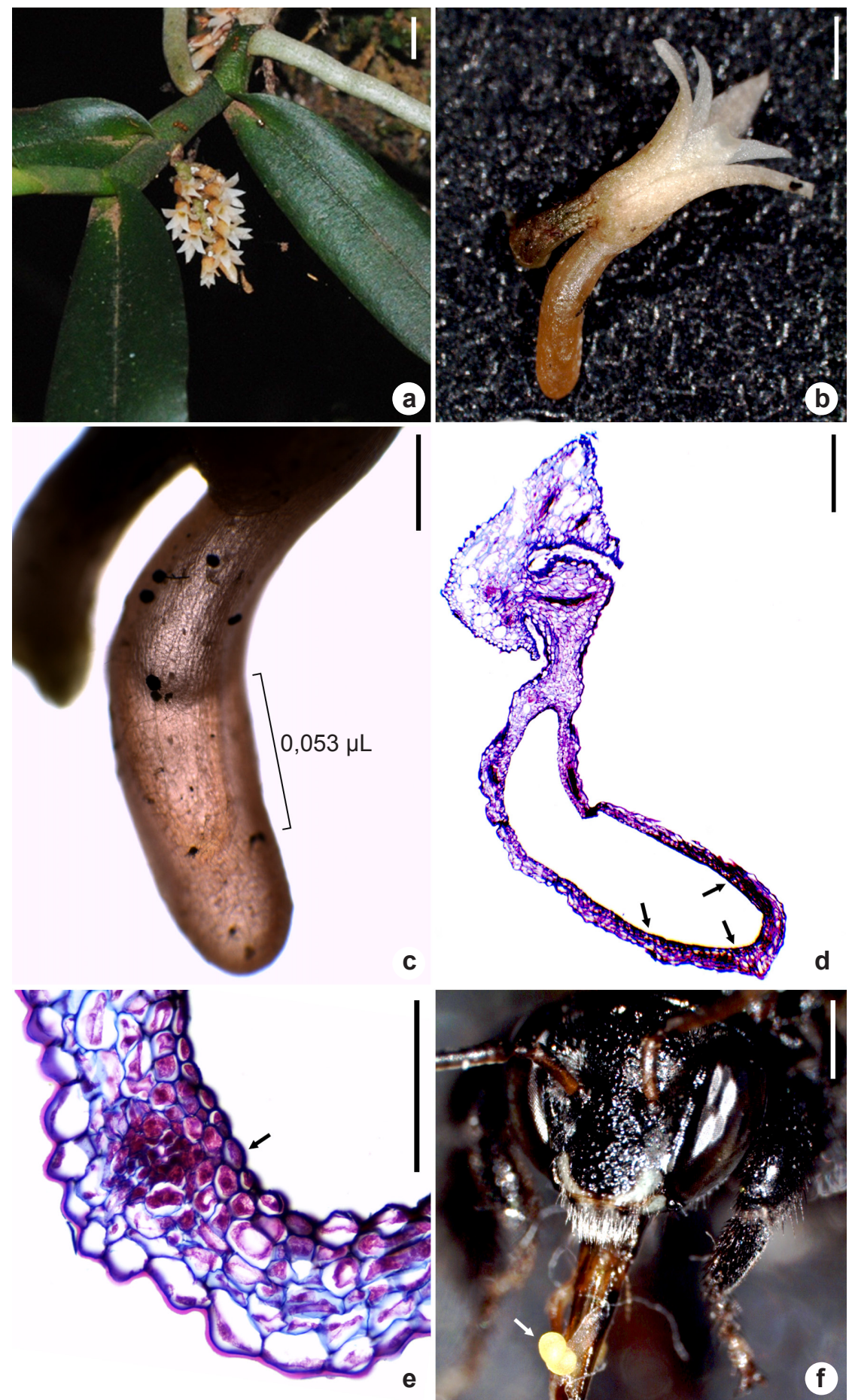

Figura 1 - Campylocentrummicranthum - a. inflorescência; b. flor em vista lateral mostrando o nectário tubular na base do labelo; c. nectário em vista lateral evidenciando a coluna de néctar; d. corte longitudinal do nectário mostrando o lúmen e a superfície secretora (setas); e. epiderme secretoras do interior do nectário (seta); f. Lophopedianigrispinis com polinário aderido às peças bucais (seta). Barras de escala: $\mathrm{a}=5 \mathrm{~mm} ; \mathrm{b}=1 \mathrm{~mm} ; \mathrm{c}-\mathrm{d}=0,5 \mathrm{~mm} ; \mathrm{e}=100 \mu \mathrm{m} ; \mathrm{f}=0,5 \mathrm{~mm}$. Figure 1 - Campylocentrummicranthum - a. inflorescence; $b$. flower in lateral view showing the tubular nectary of the base of the lip; c. detail of the nectary in lateral view evidencing the nectar column; d. longitudinal section of the nectary showing the lumen and the secretory surface (arrows); e. detail of the secretory epidermis if the inner nectary (arrow); f. Lophopedianigrispiniswith a pollinarium attached on their mouthparts (arrow). Bars: $\mathrm{a}=5 \mathrm{~mm} ; \mathrm{b}=1 \mathrm{~mm} ; \mathrm{c}-\mathrm{d}=0.5 \mathrm{~mm} ; \mathrm{e}=100 \mu \mathrm{m} ; \mathrm{f}=0.5 \mathrm{~mm}$. 
Tabela 1 - Resultados dos testes sobre o sistema de reprodução de Campylocentrummicranthum: número de frutos formados em cada um dos tratamentos realizados e em condições naturais (polinização aberta) no município de São Simão, estado de São Paulo, Brasil. Os números entre parênteses indicam a porcentagem de frutos formados.

Table 1 - Results of the reproductive system of Campylocentrummicranthum: percentage of fruit set per treatment performed and under natural conditions (open pollination) in the municipality of São Simão, state of São Paulo, southeastern Brazil. The numbers in brackets indicates the percentage of fruit set.

\begin{tabular}{lcc}
\hline Tratamento & Flores & Frutos \\
\hline Autopolinização espontânea & 82 & - \\
Autopolinização manual & 50 & $8(16 \%)$ \\
Emasculação & 30 & - \\
Polinização cruzada & 63 & $2(3,17 \%)$ \\
Condições naturais & 998 & $364(36,4 \%)$ \\
\hline
\end{tabular}

a antera, que cai espontaneamente longo em seguida. A polinização ocorreu quando uma abelha carregando um polinário visitou outra flor. Normalmente apenas uma das duas polínias foi depositada na cavidade estigmática a cada evento de polinização. Através da conferência das flores marcadas não foram detectados visitantes noturnos para indivíduos da população de Campylocentrummicranthum.

Os indivíduos estudados nessa população de Campylocentrum micranthum são autocompatíveis, porém não autógamas e necessitaram de polinizadores para a transferência do pólen entre as plantas. Os indivíduos em que as flores foram emasculadas e submetidas à autopolinização espontânea (flores intactas), não desenvolveram frutos. Em flores submetidas à polinização cruzada manual, a formação de frutos foi de $3,17 \%$, enquanto $16 \%$ das flores autopolinizadas manualmente resultaram em frutos. Isso pode estar relacionado com a limitação de recursos ou estresse causado devido à alta taxa de frutificação a que foram submetidos e também devido ao estresse causado pelo transplante dos indivíduos no processo de realização dos experimentos. Em condições naturais a população estudada apresentou uma taxa de frutificação de $36,4 \%$ (Tab. 1).

\section{Discussão}

$\mathrm{Na}$ área de estudo os indivíduos de Campylocentrum micranthum florescem no verão, entre dezembro e fevereiro, período no qual as chuvas são mais intensas no interior do estado de São Paulo e muitas espécies são encontradas em flor (Pansarin \& Pansarin 2008, 2010; Ferreira et al. 2010; Aguiar et al. 2012). Em Chiapas, no México, C. micranthum floresce entre abril e maio, e em novembro (Damon \& Salas-Roblero 2007). A ocorrência de diferenças nos períodos de floração é comum em espécies de ampla distribuição e tem sido documentada para orquídeas que ocorrem desde a América Central até o interior do estado de São Paulo (Pansarin \& Amaral 2008; Aguiar et al. 2012).

As características morfológicas aqui descritas para Campylocentrum micranthum são comumente encontradas em espécies de Angraecinae (Dressler 1981, 1993; Nilsson et al. 1985, 1987). A presença de um nectário na base do labelo, por exemplo, no qual é produzido néctar, é uma característica comumente encontrada em representantes da subtribo (Dressler 1981).

O nectário presente nas flores de Campylocentrum micranthum apresenta a epiderme lisa e as células parenquimáticas da parede interna do nectário parecem estar envolvidas no processo de secreção. Entre as orquídeas do interior do estado de São Paulo, esse mesmo padrão de tecido secretor foi documentado para Oeceoclades maculata (Lindl.) Lindl. (Aguiar et al. 2012). No entanto, em $O$. maculata o nectário apresenta tricomas na porção apical, onde o néctar fica acumulado (Aguiar et al. 2012). Em Orchidaceae, é comum que o néctar produzido seja secretado para o lúmen do nectário, assim como ocorre em C. micranthum. Todavia, algumas vezes o néctar não fica exposto, e o polinizador necessita romper a cutícula das papilas para poder acessá-lo, como documentado para Epidendrum densiflorum Hook. (como E. paniculatum Ruiz \& Pavón; Pansarin 2003). 
Baseado em características florais, membros das Angraecinae tem sido consideradas como sendo polinizados principalmente por mariposas (Dressler 1981, 1993). De fato, os primeiros estudos sobre biologia da polinização para a tribo foram realizados em Madagascar, onde o gênero Angraecum é bem representado. Darwin (1862) já havia postulado que $A$. sesquipedale Thouars seria polinizado por mariposas da família Sphingidae, baseado no comprimento do nectário, que pode chegar a $30 \mathrm{~cm}$. O provável polinizador de A. sesquipedale (Xanthopanmorgani Walker) foi descrito 41 anos após a predição de Darwin (Rothschild \& Jordan 1903). Ainda em Madagascar, Nilsson et al. $(1985,1987)$, também observaram mariposas Sphingidae atuando como polinizadoras de orquídeas deste gênero. Entretanto, não apenas mariposas são consideradas polinizadores para o gênero Angraecum. Pássaros Zosteropidae foram observados polinizando Angraecum striatum Thouars (Micheneau et al.2008b), e grilos (Orthoptera) foram documentados como polinizadores de $A$. cadetii Bosser (Micheneau et al. 2010). Como reportado aqui, as flores de Campylocentrum micranthum foram visitadas e polinizadas exclusivamente por abelhas (Lophopedia pygmaea, L. nigrispinis e Ceratina sp.) na população estudada. Em outras duas espécies estudadas no Brasil, abelhas também foram documentadas como polinizadores. Em C. aromaticum, abelhas Pseudoaugochloropisis graminea (Fabricius 1804), Agochlorella cf. eusticta Moure, Augochlora amphitrite (Schrotthy 1909) e Augochlora sp. (Hymenoptera, Halictidae) foram observadas atuando como polinizadores (Singer \& Cocucci 1999). Em Campylocentrum burchellii abelhas Trigonaspinipes (Fabricius 1793) e Plebeia amerina (Friese1900) (Apidae: Meliponini) foram observadas visitando as flores de indivíduos desta espécie (Singer \& Cocucci 1999).

As atividades dos polinizadores nas flores de Campylocentrum micranthum se iniciam por volta das $7 \mathrm{~h} 30 \mathrm{~min}$ e se estendem até às $13 \mathrm{~h}$, com as visitas ocorrendo somente em dias ensolarados. Em C. aromaticum as atividades por parte das abelhas iniciaram por volta de $8 \mathrm{~h} 45$ min e se estenderam até $13 \mathrm{~h}$, porém, com maior frequência entre $11 \mathrm{~h}$ e 13 h (Singer \& Cocucci 1999). Segundo Camargo \& Mazucato (1984), abelhas Apidae e Halictidae (Hymenoptera) normalmente apresentam um período maior de atividade entre $9 \mathrm{~h}$ e $15 \mathrm{~h}$. Este período está relacionado com temperaturas $\left(20^{\circ} \mathrm{C}-\right.$ $30^{\circ} \mathrm{C}$ ) apropriadas para atividades de forrageamento das abelhas (Linsley 1958; Hilário et al. 2001).
O comportamento das abelhas durante o processo de polinização de Campylocentrum micranthum é semelhante ao encontrado por Singer \& Cocucci (1999) para C. aromaticum. No entanto, em C. micranthum a deposição do polinário ocorre na porção dorsal da glossa e não na face ventral, como documentado para C. aromaticum (Singer \& Cocucci 1999) e em Angraecum (e.g., Nilsson et al. 1985, 1987). Durante as observações às flores de C. micranthum, foi observado que opolinário geralmente é removido junto com o capuz da antera. A retenção do capuz da antera pelas polínias é um fenômeno conhecido e tem sido documentado para várias espécies de orquídeas como um mecanismo floral que tende a evitar a ocorrência de autopolinização (e.g., Catling \& Catling 1991b; Singer \& Cocucci 1999; Borba \& Semir 2001; Mickeliunas et al. 2006).

A partir dos tratamentos para verificação dos sistemas de reprodução de Campylocentrum micranthum foi observado que a espécie é autocompatível e não autógama, uma vez que as flores tenham originado frutos somente nos tratamentos de autopolinização manual e polinização cruzada. Na subtribo Angraecinae a autocompatibilidade é uma característica comum, e tem sido documentada em várias outras espécies desse grupo (e.g., Nilsson 1988; Dressler 1993; Luyt \& Johnson 2001; Micheneau et al. 2006, 2008a,b; Martins \& Johnson 2007). Já as flores submetidas ao tratamento de emasculação ou à autopolinização espontânea não desenvolveram frutos. Geralmente, em Orchidaceae, as flores apresentam barreiras mecânicas que são eficientes para evitar a ocorrência de autopolinizações em ambiente natural (Van der Pijl \& Dodson 1966; Catling \& Catling 1991a).

No presente estudo as flores autopolinizadas e submetidas ao tratamento de polinização cruzada desenvolveram poucos frutos em relação à quantidade de flores utilizadas em cada tratamento. A alta taxa de aborto dos frutos em Campylocentrum micranthum pode estar relacionada com a limitação de recursos e estresse devido à alta taxa de frutificação a que foram submetidas às inflorescências e também devido ao transplante dos indivíduos, uma vez que os experimentos foram feitos sob o estereomicroscópio e, para que isso fosse possível, as plantas foram removidas dos vasos e novamente plantadas ao final das manipulações. Dados similares relacionados ao aborto de frutos em tratamento manuais têm sido documentados em várias outras espécies de orquídeas (e.g., Montalvo \& Ackerman 1987; Ackerman 1989; Zimmerman \& Aide 1989; Borba \& Semir 1998). 
Os dados apresentados no presente estudo mostram que a taxa de frutificação em condições naturais para Campylocentrum micranthum $(36,4 \%)$ pode ser considerada elevada quando comparados com outras espécies de orquídeas autocompatíveis e não autógamas que ocorrem no interior do estado de São Paulo. (e.g., Mickeliunas et al. 2006; Pansarin et al. 2006; Pansarin 2008; Pansarin \& Amaral 2008). Estudos têm mostrado que orquídeas, cujas flores oferecem néctar como recurso apresentam maior sucesso reprodutivo em relação às espécies que produzem qualquer outro tipo de recurso ou são polinizadas por engano (Tremblay et al. 2005). De fato, no interior do estado de São Paulo flores nectaríferas de diversas espécies, como Cyclopogon congestus (Vell.) Hoehne, Mesadenella cuspidata (Lindl.) Garay (Pansarin \& Pansarin 2010) apresentam maior taxa de frutificação em relação às espécies que oferecem outros tipos de recursos, como óleos comestíveis (e.g., Mickeliunas et al. 2006) e fragrâncias (e.g., Pansarin et al. 2006), ou são polinizadas por engano (e.g., Pansarin 2008).

\section{Agradecimentos}

Os autores agradecem à Dra. Ludimila Mickeliunas Pansarin, o auxilio na confecção das laminias para observação das células secretoras do nectário. Ao Prof. Dr. Antonio José Camilo Aguiar, da Universidade de Brasilia-UNB, por nos ajudar na identificação das abelhas. PRMC agradece ao Program de Pós-graduação em Entomologia da FFCLRP-USP e ao CNPq, a bolsa de Mestrado concedida.

\section{Referências}

Ackerman, J.D. 1989. Limitations to sexual reproduction in Encyclia krugii (Orchidaceae). Systematic Botany 14: 101-109.

Aguiar, J.M.R.B.V.; Pansarin, L.M.; Ackerman, J.D. \& Pansarin, E.R. 2012. Biotic versus abiotic pollination in Oeceoclades maculata (Lindl.) Lindl. (Orchidaceae). Plant Species Biology 27: 86-95.

Barros, F.V.; Rodrigues, F.; Barberena, V.T.; Fraga, F.F.V.A. \& Pessoa, C.N.E.M. 2012. Orchidaceae in Lista de espécies da flora do Brasil. Jardim Botânico do Rio de Janeiro. Disponível em $<$ http:// floradobrasil.jbrj.gov.br/2012/FB011267>. Acesso em 22 abril 2012.

Bogarín, D. \& Pupulin, F. 2010. The genus Campylocentrum (Orchidaceae: Agraecinae) in Costa Rica: a revision.Harvard Papers in Botany 15: 353-414.

Borba, E.L. \& Semir, J. 1998. Wind-assisted fly pollination in three Bulbophyllum (Orchidaceae) species occurring in the Brazilian campos rupestres. Lindleyana 13: 203-218.

Borba, E.L. \& Semir, J. 2001. Pollinator specificity and convergence in fly-pollinated Pleurothallis (Orchidaceae) species: a multiple population approach. Annals of Botany 88: 75-88.

Camargo, J.M.F. \& Mazucato, M. 1984. Inventário da apifauna e flora apícola da Ribeirão Preto, SP, Brasil. Dusenia 14: 55-87.

Carlsward, B.S.; Whitten, W.M. \& Willians, N.H. 2003. Molecular Phylogenetics of netropical leafless Angraecinae (Orchidaceae): Evolution of Generic Concepts 1: 43-51.

Catling, P.M. \& Catling, V.R. 1991a. A synopsis of breeding systems and pollination in North American orchids. Lindleyana 6: 187-210.

Catling, P.M. \& Catling, V.R. 1991b. Anther cap retention in Tipularia discolor. Lindleyana 6: 113-116.

CEPAGRI-Centro de Pesquisas Metereológicas e Climáticas Aplicadas a Agricultura. São Paulo. Disponível em <http://www.cpa.unicamp.br>. Acesso em 16 outubro 2012.

Damon, A. \& Salas-Roblero, P. 2007. A survey of pollination in remnant orchid populations in Soconusco, Chiapas, Mexico. Tropical Ecology 48: 1-14.

Dressler, R.L. 1981. The Orchids: natural history and classification. Harvard University Press, Harvard. $332 \mathrm{p}$.

Dressler, R.L. 1993. Phylogeny and classification of the orchid family. Timber Press, Portland. 314p.

Ferreira, A.W.C.; Lima, M.I.S. \& Pansarin, E.R. 2010. Orchidaceae na região central de São Paulo, Brasil. Rodriguésia 61:243-259.

Gerlach, G. 1969. Botanische Mikrotechnik. Georg Thieme Verlag, Stuttgard. 344p.

Hilário, S.D.; Imperatriz-Fonseca, V.L. \& Kleinert, A.M.P. 2001. Responses to climatic factors by foragers of Plebeia pugnax Moure (in Litt.) (Apidae, Meliponinae). Revista Brasileira de Biologia 61: 191-196.

Hoehne, F.C. 1949. Iconografia das orquidáceas do Brasil. Secretaria da Agricultura, São Paulo. 601p.

Johansen, D.A. 1940. Plant microtechnique. Mc Graw Hill, New York. 222p.

Köppen, W. 1948. Climatologia. Editora Fondo de Cultura Económica, México. 207p.

Linsley, E.G. 1958. The ecology of solitary bees. Hilgardia 27: 541-599.

Luyt, R. \& Johnson, S.D. 2001. Hawkmoth pollination of the African epiphytic orchid Mystacidium venosum, with special reference to flower and pollen longevity. Plant Systematics and Evolution 228: 49-62.

Martins, D.J. \& Johnson, S.D. 2007. Hawkmoth pollination of aerangoid orchids in Kenya, with special reference to nectar sugar concentration 
gradients in the floral spurs. American Journal of Botany 94: 650-659.

Mickeliunas, L.; Pansarin, E.R. \& Sazima, M. 2006. Biologia floral, melitofilia e influência de besouros Curculionidae no sucesso reprodutivo de Grobya amherstiae Lindl. (Orchidaceae: Cyrtopodiinae). Revista Brasileira de Botânica 29: 251-258.

Micheneau, C.; Fournel, J. \& Pailler, T. 2006. Bird pollination in an angraecoid orchid on Reunion Island (Mascarene Archipelago, Indian Ocean). Annals of Botany 97: 965-974.

Micheneau, C.; Fournel, J.; Gauvin-Bialecki, A. \& Pailler, T. 2008a. Auto-pollination in a long-spurred endemic orchid (Jumellea stenophylla) on Reunion Island (Mascarene Archipelago, Indian Ocean). Plant Systematics and Evolution 272: 11-22.

Micheneau, C.; Fournel, J.; Humeau, L. \& Pailler, T. 2008b. Orchid-bird interactions: a case study from Angraecum (Vandeae, Angraecinae) and Zosterops (white-eyes, Zosteropidae) on Reunion Island. Botany 86: 1143-1151.

Micheneau, C.; Fournel, J.; Warren, B.H.; Hugel, S.; Gauvin-Bialecki, A.; Pailler, T.; Strasberg, D. \& Chase, M.W. 2010. Orthoptera, a new order of pollinator. Annals of Botany 105: 355-364.

Montalvo, A.M. \& Ackerman, J.D. 1987. Limitations to fruit production in Ionopsis utricularioides (Orchidaceae). Biotropica 19: 24-31.

Nilsson, L.A.; Jonsson, L.; Ralison, L. \& Randrianjohany, E. 1985. Monophily and pollination mechanisms in Angraecum arachnites Shltr. (Orchidaceae) in a guild of long-tongued hawk-moths (Sphingidae) in Madagascar. Biological Journal of the Linnean Society 91: 1-19.

Nilsson, L.A.; Jonsson, L.; Ralison, L. \& Randrianjohany, E. 1987. Angraecoid orchids and hawk-moths in central Madagascar: specialized pollination systems and generalist foragers. Biotropica 19: 310-318.

Nilsson, L.A. 1988. The evolution of flowers with deep corolla tubes. Nature 334: 147-149.

Pabst, G.F.J. \& Dungs, F. 1977. Orchidaceae brasilienses. Band II. Kurt Schmersow, Hildesheim. 418p.

Pansarin, E.R. 2003. Biologia reprodutiva e polinização em Epidendrum paniculatum Ruiz \& Pavón
(Orchidaceae). Revista Brasileira de Botânica 26: 203-211.

Pansarin, E.R.; Bittrich, V. \& Amaral, M.C.E. 2006. At daybreak reproductive biology and isolating mechanisms of Cirrhaea dependens (Orchidaceae). Plant Biology 8: 494-502.

Pansarin, E.R. \& Amaral, M.C.E. 2008. Reproductive biology and pollination mechanisms of Epidendrum secundum (Orchidaceae). Floral variation: a consequence of natural hybridization? Plant Biology 10: 211-219.

Pansarin, E.R. \& Pansarin, L.M. 2008. A família Orchidaceae na Serra do Japi, São Paulo, Brasil. Rodriguésia 59: 99-111.

Pansarin, E.R. 2008. Reproductive biology and pollination of Govenia utriculata: a syrphid fly orchid pollinated through a pollen deceptive mechanism. Plant Species Biology 23: 90-96.

Pansarin, E.R. \& Pansarin, L.M. 2010. The family Orchidaceae in the Serra do Japi, state of São Paulo, Brazil. Springer Wien New York, Wien. 290p.

Rothschild, L.W. \& Jordan, K. 1903. A revision of the lepidopterous family Sphingidae. Novitates Zoological 9: 1-972.

Singer, R.B. \& Coccuci, A.A. 1999. Pollination mechanism in southern Brazilian orchids which are exclusively or mainly pollinated by Halictid bees. Plant Systematics and Evolution 217: 101-117.

Todzia, C.A. 1980. A revision of the Costa Rican species of Campylocentrum (Orchidaceae) Brenesia 18: 117-136.

Tremblay, R.L.; Ackerman, J.D.; Zimmerman, J.K. \& Calvo, R.N. 2005. Variation in sexual reproduction in orchids and its evolutionary consequences: a spasmodic journey to diversification. Biological Journal of Linnean Society 84: 1-54.

Van Der Pijl \& Dodson, C.H. 1966. Orchid flowers, their pollination and evolution. University of Miami Press, Florida. 214p.

Zimmerman, J.K. \& Aide, T.M. 1989. Patterns of fruit production in a neotropical orchid: pollinator vs. Resource limitation. American Journal of Botany 76: $67-73$. 